\title{
FSIM: A Feature Similarity Index for Image Quality Assessment
}

\author{
Lin Zhang ${ }^{\mathrm{a}}$, Student Member, IEEE, Lei Zhang ${ }^{\mathrm{a}, 1}$, Member, IEEE \\ Xuanqin Mou ${ }^{\mathrm{b}}$, Member, IEEE, and David Zhang ${ }^{\mathrm{a}}$, Fellow, IEEE \\ ${ }^{\mathrm{a} D e p a r t m e n t ~ o f ~ C o m p u t i n g, ~ T h e ~ H o n g ~ K o n g ~ P o l y t e c h n i c ~ U n i v e r s i t y, ~ H o n g ~ K o n g ~}$ \\ ${ }^{\mathrm{b}}$ Institute of Image Processing and Pattern Recognition, Xi'an Jiaotong University, China
}

\begin{abstract}
Image quality assessment (IQA) aims to use computational models to measure the image quality consistently with subjective evaluations. The well-known structural-similarity (SSIM) index brings IQA from pixel-based stage to structure-based stage. In this paper, a novel feature-similarity (FSIM) index for full reference IQA is proposed based on the fact that human visual system (HVS) understands an image mainly according to its low-level features. Specifically, the phase congruency (PC), which is a dimensionless measure of the significance of a local structure, is used as the primary feature in FSIM. Considering that PC is contrast invariant while the contrast information does affect HVS' perception of image quality, the image gradient magnitude (GM) is employed as the secondary feature in FSIM. PC and GM play complementary roles in characterizing the image local quality. After obtaining the local quality map, we use PC again as a weighting function to derive a single quality score. Extensive experiments performed on six benchmark IQA databases demonstrate that FSIM can achieve much higher consistency with the subjective evaluations than state-of-the-art IQA metrics.
\end{abstract}

Index Terms: Image quality assessment, phase congruency, gradient, low-level feature

\section{INTRODUCTION}

With the rapid proliferation of digital imaging and communication technologies, image quality assessment (IQA) has been becoming an important issue in numerous applications such as image acquisition, transmission, compression, restoration and enhancement, etc. Since the subjective IQA methods cannot be

\footnotetext{
${ }^{1}$ Corresponding author. Email: cslzhang@comp.polyu.edu.hk. This project is supported by the Hong Kong RGC General Research Fund (PolyU 5330/07E), the Ho Tung Fund (5-ZH25) and and NSFC 90920003.
} 
readily and routinely used for many scenarios, e.g. real-time and automated systems, it is necessary to develop objective IQA metrics to automatically and robustly measure the image quality. Meanwhile, it is anticipated that the evaluation results should be statistically consistent with those of the human observers. To this end, the scientific community has developed various IQA methods in the past decades. According to the availability of a reference image, objective IQA metrics can be classified as full reference (FR), no-reference (NR) and reduced-reference (RR) methods [1]. In this paper, the discussion is confined to FR methods, where the original "distortion free" image is known as the reference image.

The conventional metrics such as the peak signal-to-noise ratio (PSNR) and the mean squared error (MSE) operate directly on the intensity of the image and they do not correlate well with the subjective fidelity ratings. Thus many efforts have been made on designing human visual system (HVS) based IQA metrics. Such kinds of models emphasize the importance of HVS' sensitivity to different visual signals, such as the luminance, the contrast, the frequency content, and the interaction between different signal components [2-4]. The noise quality measure (NQM) [2] and the visual signal-to-noise ratio (VSNR) [3] are two representatives. Methods such as the structural similarity (SSIM) index [1] are motivated by the need to capture the loss of structure in the image. SSIM is based on the hypothesis that HVS is highly adapted to extract the structural information from the visual scene; therefore, a measurement of structural similarity should provide a good approximation of perceived image quality. The multi-scale extension of SSIM, called MS-SSIM [5], produces better results than its single-scale counterpart. In [6], the authors presented a 3-component weighted SSIM (3-SSIM) by assigning different weights to the SSIM scores according to the local region type: edge, texture or smooth area. In [7], Sheikh et al. introduced the information theory into image fidelity measurement, and proposed the information fidelity criterion (IFC) for IQA by quantifying the information shared between the distorted and the reference images. IFC was later extended to the visual information fidelity (VIF) metric in [4]. In [8], Sampat et al. made use of the steerable complex wavelet transform to measure the structural similarity of the two images and proposed the CW-SSIM index.

Recent studies conducted in [9] and [10] have demonstrated that SSIM, MS-SSIM, and VIF could offer statistically much better performance in predicting images' fidelity than the other IQA metrics. However, SSIM and MS-SSIM share a common deficiency that when pooling a single quality score from the local quality map (or the local distortion measurement map), all positions are considered to have the same importance. In VIF, images are decomposed in different sub-bands and these sub-bands can have different 
weights at the pooling stage [11]; however, within each sub-band, every position is still given the same importance. Such pooling strategies are not consistent with the intuition that different locations on an image can have very different contributions to HVS' perception of the image. This is corroborated by a recent study $[12,13]$, where the authors found that by incorporating appropriate spatially varying weights, the performance of some IQA metrics, e.g., SSIM, VIF, and PSNR, could be improved. But unfortunately, they did not present an automated method to generate such weights.

The great success of SSIM and its extensions owes to the fact that HVS is adapted to the structural information in images. The visual information in an image, however, is often very redundant, while the HVS understands an image mainly based on its low-level features, such as edges and zero-crossings [14-16]. In other words, the salient low-level features convey crucial information for the HVS to interpret the scene. Accordingly, perceptible image degradations will lead to perceptible changes in image low-level features, and hence a good IQA metric could be devised by comparing the low-level feature sets between the reference image and the distorted image. Based on the above analysis, in this paper we propose a novel low-level feature similarity induced FR IQA metric, namely FSIM (Feature SIMilarity).

One key issue is then what kinds of features could be used in designing FSIM? Based on the physiological and psychophysical evidence, it is found that visually discernable features coincide with those points where the Fourier waves at different frequencies have congruent phases [16-19]. That is, at points of high phase congruency (PC) we can extract highly informative features. Such a conclusion has been further corroborated by some recent studies in neurobiology using functional magnetic resonance imaging (fMRI) [20]. Therefore, PC is used as the primary feature in computing FSIM. Meanwhile, considering that PC is contrast invariant but image local contrast does affect HVS' perception on the image quality, the image gradient magnitude (GM) is computed as the secondary feature to encode contrast information. PC and GM are complementary and they reflect different aspects of the HVS in assessing the local quality of the input image. After computing the local similarity map, PC is utilized again as a weighting function to derive a single similarity score. Although FSIM is designed for grayscale images (or the luminance components of color images), the chrominance information can be easily incorporated by means of a simple extension of FSIM, and we call this extension FSIM $_{C}$.

Actually, PC has already been used for IQA in the literature. In [21], Liu and Laganière proposed a PC-based IQA metric. In their method, PC maps are partitioned into sub-blocks of size $5 \times 5$. Then, the cross 
correlation is used to measure the similarity between two corresponding PC sub-blocks. The overall similarity score is obtained by averaging the cross correlation values from all block pairs. In [22], PC was extended to phase coherence which can be used to characterize the image blur. Based on [22], Hassen et al. proposed an NR IQA metric to assess the sharpness of an input image [23].

The proposed FSIM and SSIM $_{C}$ are evaluated on six benchmark IQA databases in comparison with eight state-of-the-art IQA methods. The extensive experimental results show that FSIM and $\mathrm{FSIM}_{C}$ can achieve very high consistency with human subjective evaluations, outperforming all the other competitors. Particularly, FSIM and FSIM $_{C}$ work consistently well across all the databases, while other methods may work well only on some specific databases. To facilitate repeatable experimental verifications and comparisons, the Matlab source code of the proposed FSIM/FSIM $C$ indices and our evaluation results are available online at http://www.comp.polyu.edu.hk/ cslzhang/IQA/FSIM/FSIM.htm.

The remainder of this paper is organized as follows. Section II discusses the extraction of PC and GM. Section III presents in detail the computation of the FSIM and FSIM $_{C}$ indices. Section IV reports the experimental results. Finally, Section V concludes the paper.

\section{EXTRACTION OF PHASE CONGRUENCY AND GRADIENT MAGNITUDE}

\section{A. Phase congruency (PC)}

Rather than define features directly at points with sharp changes in intensity, the PC model postulates that features are perceived at points where the Fourier components are maximal in phase. Based on the physiological and psychophysical evidences, the PC theory provides a simple but biologically plausible model of how mammalian visual systems detect and identify features in an image [16-20]. PC can be considered as a dimensionless measure for the significance of a local structure.

Under the definition of PC in [17], there can be different implementations to compute the PC map of a given image. In this paper we adopt the method developed by Kovesi in [19], which is widely used in literature. We start from the 1D signal $g(x)$. Denote by $M_{n}^{e}$ and $M_{n}^{o}$ the even-symmetric and odd-symmetric filters on scale $n$ and they form a quadrature pair. Responses of each quadrature pair to the signal will form a response vector at position $x$ on scale $n:\left[e_{n}(x), o_{n}(x)\right]=\left[g(x)^{*} M_{n}^{e}, g(x)^{*} M_{n}^{o}\right]$, and the local amplitude on 
scale $n$ is $A_{n}(x)=\sqrt{e_{n}(x)^{2}+o_{n}(x)^{2}}$. Let $F(x)=\sum_{n} e_{n}(x)$ and $H(x)=\sum_{n} o_{n}(x)$. The 1D PC can be computed as

$$
P C(x)=E(x) /\left(\varepsilon+\sum_{n} A_{n}(x)\right)
$$

where $E(x)=\sqrt{F^{2}(x)+H^{2}(x)}$ and $\varepsilon$ is a small positive constant.

With respect to the quadrature pair of filters, i.e. $M_{n}^{e}$ and $M_{n}^{o}$, Gabor filters [24] and log-Gabor filters [25] are two widely used candidates. We adopt the log-Gabor filters because: 1) one cannot construct Gabor filters of arbitrarily bandwidth and still maintain a reasonably small DC component in the even-symmetric filter, while log-Gabor filters, by definition, have no DC component; and 2) the transfer function of the log-Gabor filter has an extended tail at the high frequency end, which makes it more capable to encode natural images than ordinary Gabor filters $[19,25]$. The transfer function of a log-Gabor filter in the frequency domain is $G(\omega)=\exp \left(-\left(\log \left(\omega / \omega_{0}\right)\right)^{2} / 2 \sigma_{r}^{2}\right)$, where $\omega_{0}$ is the filter's center frequency and $\sigma_{r}$ controls the filter's bandwidth.

To compute the PC of 2D grayscale images, we can apply the 1D analysis over several orientations and then combine the results using some rule. The 1D log-Gabor filters described above can be extended to 2D ones by simply applying some spreading function across the filter perpendicular to its orientation. One widely used spreading function is Gaussian [19, 26-28]. According to [19], there are some good reasons to choose Gaussian. Particularly, the phase of any function would stay unaffected after being smoothed with Gaussian. Thus, the phase congruency would be preserved. By using Gaussian as the spreading function, the 2D log-Gabor function has the following transfer function

$$
G_{2}\left(\omega, \theta_{j}\right)=\exp \left(-\frac{\left(\log \left(\omega / \omega_{0}\right)\right)^{2}}{2 \sigma_{r}^{2}}\right) \cdot \exp \left(-\frac{\left(\theta-\theta_{j}\right)^{2}}{2 \sigma_{\theta}^{2}}\right)
$$

where $\theta_{j}=j \pi / J, j=\{0,1, \ldots, J-1\}$ is the orientation angle of the filter, $J$ is the number of orientations and $\sigma_{\theta}$ determines the filter's angular bandwidth. An example of the 2D log-Gabor filter in the frequency domain, with $\omega_{0}=1 / 6, \theta_{j}=0, \sigma_{r}=0.3$, and $\sigma_{\theta}=0.4$, is shown in Fig. 1 .

By modulating $\omega_{0}$ and $\theta_{j}$ and convolving $G_{2}$ with the 2D image, we get a set of responses at each point $\mathbf{x}$ as $\left[e_{n, \theta_{j}}(\mathbf{x}), o_{n, \theta_{j}}(\mathbf{x})\right]$. The local amplitude on scale $n$ and orientation $\theta_{j}$ is $A_{n, \theta_{j}}(\mathbf{x})=\sqrt{e_{n, \theta_{j}}(\mathbf{x})^{2}+o_{n, \theta_{j}}(\mathbf{x})^{2}}$, and the local energy along orientation $\theta_{j}$ is $E_{\theta_{j}}(\mathbf{x})=\sqrt{F_{\theta_{j}}(\mathbf{x})^{2}+H_{\theta_{j}}(\mathbf{x})^{2}}$, where $F_{\theta_{j}}(\mathbf{x})=\sum_{n} e_{n, \theta_{j}}(\mathbf{x})$ and $H_{\theta_{j}}(\mathbf{x})=\sum_{n} o_{n, \theta_{j}}(\mathbf{x})$. The 2D PC at $\mathbf{x}$ is defined as 


$$
P C_{2 D}(\mathbf{x})=\frac{\sum_{j} E_{\theta_{j}}(\mathbf{x})}{\varepsilon+\sum_{n} \sum_{j} A_{n, \theta_{j}}(\mathbf{x})}
$$

It should be noted that $P C_{2 D}(\mathbf{x})$ is a real number within $0 \sim 1$. Examples of the PC maps of $2 \mathrm{D}$ images can be found in Fig. 2.

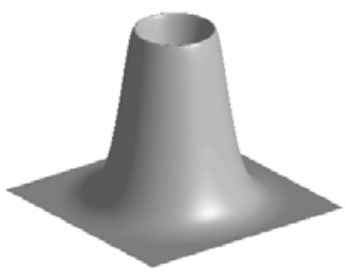

(a)

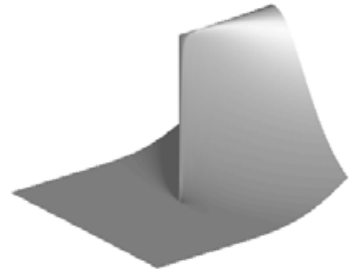

(b)

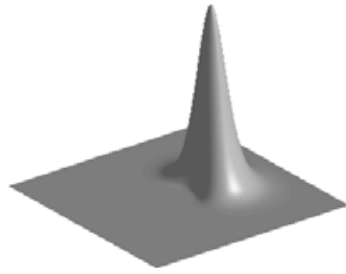

(c)

Fig. 1: An example of the log-Gabor filter in the frequency domain, with $\omega_{0}=1 / 6, \theta_{j}=0, \sigma_{r}=0.3$, and $\sigma_{\theta}=0.4$. (a) The radial component of the filter. (b) The angular component of the filter. (c) The log-Gabor filter, which is the product of the radial component and the angular component.

\section{B. Gradient magnitude (GM)}

Image gradient computation is a traditional topic in image processing. Gradient operators can be expressed by convolution masks. Three commonly used gradient operators are the Sobel operator [29], the Prewitt operator [29] and the Scharr operator [30]. Their performances will be examined in the section of experimental results. The partial derivatives $G_{x}(\mathbf{x})$ and $G_{y}(\mathbf{x})$ of the image $f(\mathbf{x})$ along horizontal and vertical directions using the three gradient operators are listed in Table I. The gradient magnitude (GM) of $f(\mathbf{x})$ is then defined as $G=\sqrt{G_{x}^{2}+G_{y}^{2}}$.

TABLE I. PARTIAL DERIVATIVES OF $f(\mathbf{x})$ USING DIFFERENT GRADIENT OPERATORS

\begin{tabular}{lccc}
\hline & Sobel & Prewitt & Scharr \\
\hline$G_{x}(\mathbf{x})$ & $\frac{1}{4}\left[\begin{array}{ccc}1 & 0 & -1 \\
2 & 0 & -2 \\
1 & 0 & -1\end{array}\right] * f(\mathbf{x})$ & $\frac{1}{3}\left[\begin{array}{ccc}1 & 0 & -1 \\
1 & 0 & -1 \\
1 & 0 & -1\end{array}\right] * f(\mathbf{x})$ & $\frac{1}{16}\left[\begin{array}{ccc}3 & 0 & -3 \\
10 & 0 & -10 \\
3 & 0 & -3\end{array}\right] * f(\mathbf{x})$ \\
$G_{y}(\mathbf{x})$ & $\frac{1}{4}\left[\begin{array}{ccc}1 & 2 & 1 \\
0 & 0 & 0 \\
-1 & -2 & -1\end{array}\right] * f(\mathbf{x})$ & $\frac{1}{3}\left[\begin{array}{ccc}1 & 1 & 1 \\
0 & 0 & 0 \\
-1 & -1 & -1\end{array}\right] * f(\mathbf{x})$ & $\frac{1}{16}\left[\begin{array}{ccc}3 & 10 & 3 \\
0 & 0 & 0 \\
-3 & -10 & -3\end{array}\right] * f(\mathbf{x})$ \\
\hline
\end{tabular}




\section{THE FEATURE SIMILARITY (FSIM) INDEX}

With the extracted PC and GM feature maps, in this section we present a novel Feature SIMilarity (FSIM) index for IQA. Suppose that we are going to calculate the similarity between images $f_{1}$ and $f_{2}$. Denote by $P C_{1}$ and $P C_{2}$ the PC maps extracted from $f_{1}$ and $f_{2}$, and $G_{1}$ and $G_{2}$ the GM maps extracted from them. It should be noted that for color images, PC and GM features are extracted from their luminance channels. FSIM will be defined and computed based on $P C_{1}, P C_{2}, G_{1}$ and $G_{2}$. Furthermore, by incorporating the image chrominance information into FSIM, an IQA index for color images, denoted by FSIM $_{C}$, will be obtained.

\section{A. The FSIM index}

The computation of FSIM index consists of two stages. In the first stage, the local similarity map is computed, and then in the second stage, we pool the similarity map into a single similarity score.

We separate the feature similarity measurement between $f_{1}(\mathbf{x})$ and $f_{2}(\mathbf{x})$ into two components, each for $\mathrm{PC}$ or GM. First, the similarity measure for $P C_{1}(\mathbf{x})$ and $P C_{2}(\mathbf{x})$ is defined as

$$
S_{P C}(\mathbf{x})=\frac{2 P C_{1}(\mathbf{x}) \cdot P C_{2}(\mathbf{x})+T_{1}}{P C_{1}^{2}(\mathbf{x})+P C_{2}^{2}(\mathbf{x})+T_{1}}
$$

where $T_{1}$ is a positive constant to increase the stability of $S_{P C}$ (such a consideration was also included in SSIM [1]). In practice, the determination of $T_{1}$ depends on the dynamic range of PC values. Eq. (4) is a commonly used measure to define the similarity of two positive real numbers [1] and its result ranges within $(0,1]$. Similarly, the GM values $G_{1}(\mathbf{x})$ and $G_{2}(\mathbf{x})$ are compared and the similarity measure is defined as

$$
S_{G}(\mathbf{x})=\frac{2 G_{1}(\mathbf{x}) \cdot G_{2}(\mathbf{x})+T_{2}}{G_{1}^{2}(\mathbf{x})+G_{2}^{2}(\mathbf{x})+T_{2}}
$$

where $T_{2}$ is a positive constant depending on the dynamic range of GM values. In our experiments, both $T_{1}$ and $T_{2}$ will be fixed to all databases so that the proposed FSIM can be conveniently used. Then, $S_{P C}(\mathbf{x})$ and $S_{G}(\mathbf{x})$ are combined to get the similarity $S_{L}(\mathbf{x})$ of $f_{1}(\mathbf{x})$ and $f_{2}(\mathbf{x})$. We define $S_{L}(\mathbf{x})$ as

$$
S_{L}(\mathbf{x})=\left[S_{P C}(\mathbf{x})\right]^{\alpha} \cdot\left[S_{G}(\mathbf{x})\right]^{\beta}
$$

where $\alpha$ and $\beta$ are parameters used to adjust the relative importance of PC and GM features. In this paper, we set $\alpha=\beta=1$ for simplicity. Thus, $S_{L}(\mathbf{x})=\mathrm{S}_{P C}(\mathbf{x}) \cdot \mathrm{S}_{G}(\mathbf{x})$.

Having obtained the similarity $S_{L}(\mathbf{x})$ at each location $\mathbf{x}$, the overall similarity between $f_{1}$ and $f_{2}$ can be 
calculated. However, different locations have different contributions to HVS' perception of the image. For example, edge locations convey more crucial visual information than the locations within a smooth area. Since human visual cortex is sensitive to phase congruent structures [20], the PC value at a location can reflect how likely it is a perceptibly significant structure point. Intuitively, for a given location $\mathbf{x}$, if anyone of $f_{1}(\mathbf{x})$ and $f_{2}(\mathbf{x})$ has a significant $\mathrm{PC}$ value, it implies that this position $\mathbf{x}$ will have a high impact on HVS in evaluating the similarity between $f_{1}$ and $f_{2}$. Therefore, we use $P C_{m}(\mathbf{x})=\max \left(P C_{1}(\mathbf{x}), P C_{2}(\mathbf{x})\right)$ to weight the importance of $S_{L}(\mathbf{x})$ in the overall similarity between $f_{1}$ and $f_{2}$, and accordingly the FSIM index between $f_{1}$ and $f_{2}$ is defined as

$$
\mathrm{FSIM}=\frac{\sum_{\mathbf{x} \in \Omega} S_{L}(\mathbf{x}) \cdot P C_{m}(\mathbf{x})}{\sum_{\mathbf{x} \in \Omega} P C_{m}(\mathbf{x})}
$$

where $\Omega$ means the whole image spatial domain.

\section{B. Extension to color image quality assessment}

The FSIM index is designed for grayscale images or the luminance components of color images. Since the chrominance information will also affect HVS in understanding the images, better performance can be expected if the chrominance information is incorporated in FSIM for color IQA. Such a goal can be achieved by applying a straightforward extension to the FSIM framework.

At first, the original RGB color images are converted into another color space, where the luminance can be separated from the chrominance. To this end, we adopt the widely used YIQ color space [31], in which $Y$ represents the luminance information and $I$ and $Q$ convey the chrominance information. The transform from the RGB space to the YIQ space can be accomplished via [31]:

$$
\left[\begin{array}{l}
Y \\
I \\
Q
\end{array}\right]=\left[\begin{array}{rrr}
0.299 & 0.587 & 0.114 \\
0.596 & -0.274 & -0.322 \\
0.211 & -0.523 & 0.312
\end{array}\right]\left[\begin{array}{l}
R \\
G \\
B
\end{array}\right]
$$

Let $I_{1}\left(I_{2}\right)$ and $Q_{1}\left(Q_{2}\right)$ be the $I$ and $Q$ chromatic channels of the image $f_{1}\left(f_{2}\right)$, respectively. Similar to the definitions of $S_{P C}(\mathbf{x})$ and $S_{G}(\mathbf{x})$, we define the similarity between chromatic features as

$$
S_{I}(\mathbf{x})=\frac{2 I_{1}(\mathbf{x}) \cdot I_{2}(\mathbf{x})+T_{3}}{I_{1}^{2}(\mathbf{x})+I_{2}^{2}(\mathbf{x})+T_{3}}, \quad S_{Q}(\mathbf{x})=\frac{2 Q_{1}(\mathbf{x}) \cdot Q_{2}(\mathbf{x})+T_{4}}{Q_{1}^{2}(\mathbf{x})+Q_{2}^{2}(\mathbf{x})+T_{4}}
$$




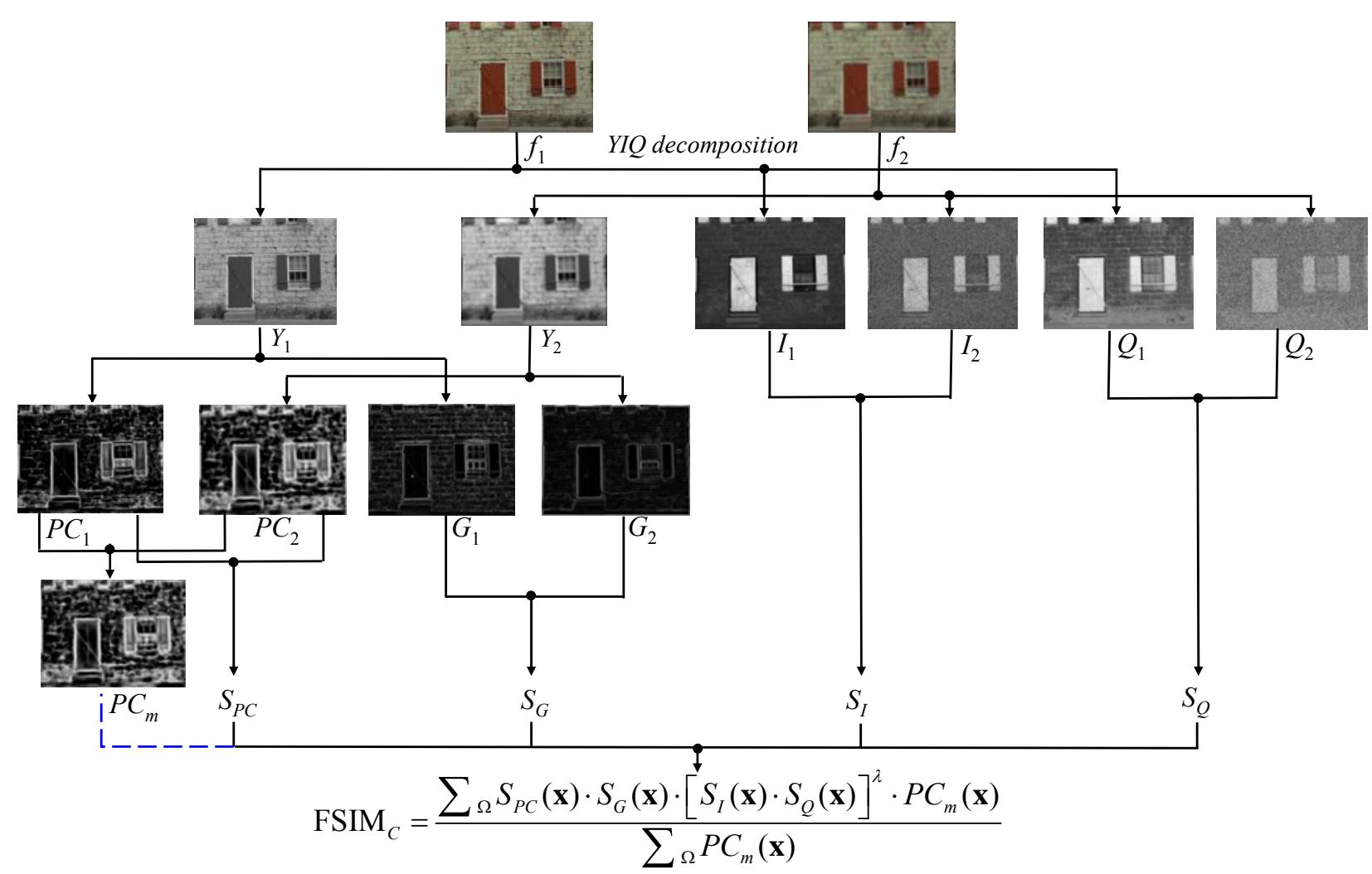

Fig. 2: Illustration for the FSIM/FSIM ${ }_{C}$ index computation. $f_{1}$ is the reference image and $f_{2}$ is a distorted version of $f_{1}$.

where $T_{3}$ and $T_{4}$ are positive constants. Since $I$ and $Q$ components have nearly the same dynamic range, in this paper we set $T_{3}=T_{4}$ for simplicity. $S_{I}(\mathbf{x})$ and $S_{Q}(\mathbf{x})$ can then be combined to get the chrominance similarity measure, denoted by $S_{C}(\mathbf{x})$, of $f_{1}(\mathbf{x})$ and $f_{2}(\mathbf{x})$ :

$$
S_{C}(\mathbf{x})=S_{I}(\mathbf{x}) \cdot S_{Q}(\mathbf{x})
$$

Finally, the FSIM index can be extended to FSIM $_{C}$ by incorporating the chromatic information in a straightforward manner:

$$
\mathrm{FSIM}_{C}=\frac{\sum_{\mathbf{x} \in \Omega} S_{L}(\mathbf{x}) \cdot\left[S_{C}(\mathbf{x})\right]^{\lambda} \cdot P C_{m}(\mathbf{x})}{\sum_{\mathbf{x} \in \Omega} P C_{m}(\mathbf{x})}
$$

where $\lambda>0$ is the parameter used to adjust the importance of the chromatic components. The procedures to calculate the FSIM/FSIM $C$ indices are illustrated in Fig. 2. If the chromatic information is ignored in Fig. 2, the $\mathrm{FSIM}_{C}$ index is reduced to the FSIM index.

\section{EXPERIMENTAL RESULTS AND DISCUSSIONS}

\section{A. Databases and methods for comparison}

To the best of our knowledge, there are six publicly available image databases in the IQA community, 
including TID2008 [10], CSIQ [32], LIVE [33], IVC [34], MICT [35] and A57 [36]. All of them will be used here for algorithm validation and comparison. The characteristics of these six databases are summarized in Table II.

TABLE II. BENCHMARK TEST DATABASES FOR IQA

\begin{tabular}{cccccc}
\hline Database & Source Images & Distorted Images & Distortion Types & Image Type & Observers \\
\hline TID2008 & 25 & 1700 & 17 & color & 838 \\
CSIQ & 30 & 866 & 6 & color & 35 \\
LIVE & 29 & 779 & 5 & color & 161 \\
IVC & 10 & 185 & 4 & color & 15 \\
MICT & 14 & 168 & 2 & color & 16 \\
A57 & 3 & 54 & 6 & gray & unknown \\
\hline
\end{tabular}

The performance of the proposed FSIM and FSIM $_{C}$ indices will be evaluated and compared with eight representative IQA metrics, including seven state-of-the-arts (SSIM [1], MS-SSIM [5], VIF [4], VSNR [3], IFC [7], NQM [2], and Liu et al's method [21]) and the classical PSNR. For Liu et al's method [21], we implemented it by ourselves. For SSIM [1], we used the implementation provided by the author, which is available at [37]. For all the other methods evaluated, we used the public software MeTriX MuX [38]. The Matlab source code of the proposed FSIM/FSIM ${ }_{C}$ indices is available online at http://www.comp.polyu.edu.hk/ cslzhang/IQA/FSIM/FSIM.htm.

Four commonly used performance metrics are employed to evaluate the competing IQA metrics. The first two are the Spearman rank-order correlation coefficient (SROCC) and the Kendall rank-order correlation coefficient (KROCC), which can measure the prediction monotonicity of an IQA metric. These two metrics operate only on the rank of the data points and ignore the relative distance between data points. To compute the other two metrics we need to apply a regression analysis, as suggested by the video quality experts group (VQEG) [39], to provide a nonlinear mapping between the objective scores and the subjective mean opinion scores (MOS). The third metric is the Pearson linear correlation coefficient (PLCC) between MOS and the objective scores after nonlinear regression. The fourth metric is the root mean squared error (RMSE) between MOS and the objective scores after nonlinear regression. For the nonlinear regression, we used the following mapping function [9]:

$$
f(x)=\beta_{1}\left(\frac{1}{2}-\frac{1}{1+e^{\beta_{2}\left(x-\beta_{3}\right)}}\right)+\beta_{4} x+\beta_{5}
$$


where $\beta_{i}, i=1,2, \ldots, 5$, are the parameters to be fitted. A better objective IQA measure is expected to have higher SROCC, KROCC and PLCC while lower RMSE values.

\section{B. Determination of parameters}

There are several parameters need to be determined for FSIM and FSIM $_{C}$. To this end, we tuned the parameters based on a sub-dataset of TID2008 database, which contains the first 8 reference images in TID2008 and the associated 544 distorted images. The 8 reference images used in the tuning process are shown in Fig. 3. The tuning criterion was that the parameter value leading to a higher SROCC would be chosen. As a result, the parameters required in the proposed methods were set as: $n=4, J=4, \sigma_{r}=0.5978, \sigma_{\theta}$ $=0.6545, T_{1}=0.85, T_{2}=160, T_{3}=T_{4}=200$, and $\lambda=0.03$. Besides, the center frequencies of the log-Gabor filters at four scales were set as: 1/6, 1/12, 1/24 and 1/48. These parameters were then fixed for all the following experiments conducted. In fact, we have also used the last 8 reference images (and the associated 544 distorted ones) to tune parameters and obtained very similar parameters to the ones reported here. This may imply that any 8 reference images in the TID2008 database work equally well in tuning parameters for

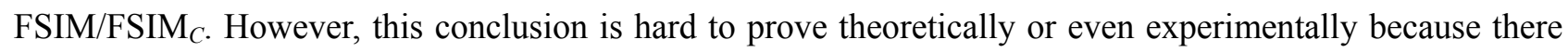
are $\mathrm{C}_{25}^{8}=1081575$ different ways to select 8 out of the 25 reference images in TID2008.

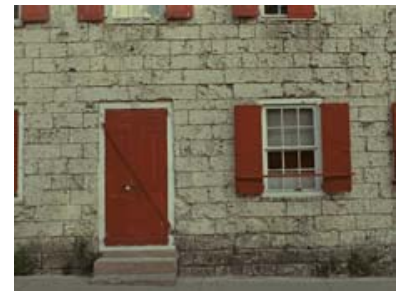

(a)

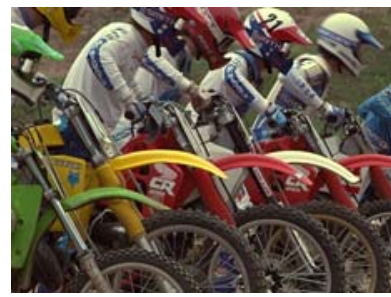

(e)

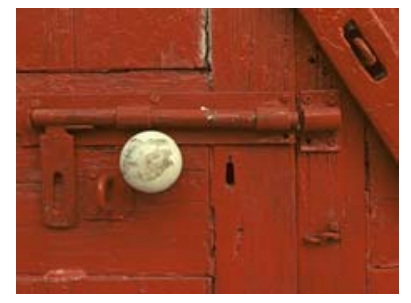

(b)

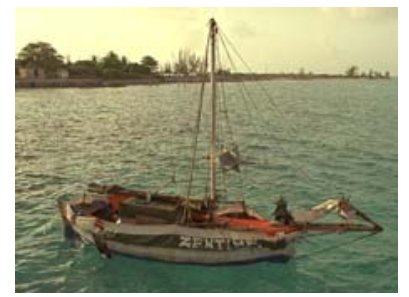

(f)

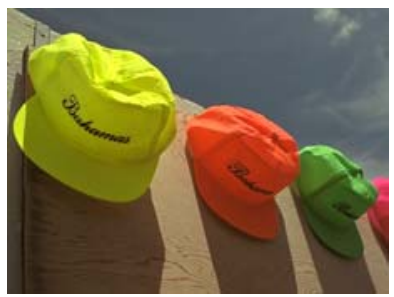

(c)

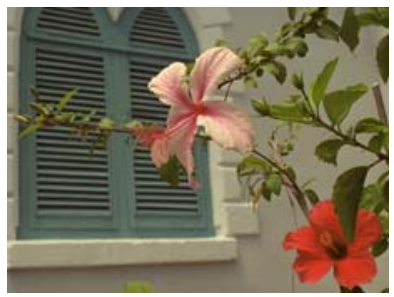

(g)

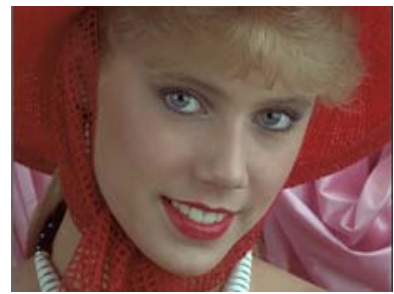

(d)

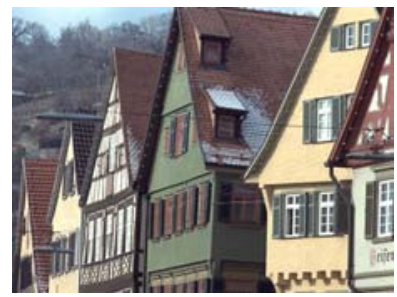

(h)

Fig. 3: Eight reference images used for the parameter tuning process. They are extracted from the TID2008 database.

It should be noted that the FSIM/FSIM $C$ indices will be most effective if used on the appropriate scale. 
The precisely "right" scale depends on both the image resolution and the viewing distance and hence is difficult to be obtained. In practice, we used the following empirical steps proposed by Wang [37] to determine the scale for images viewed from a typical distance: 1$)$ let $F=\max (1, \operatorname{round}(N / 256))$, where $N$ is the number of pixels in image height or width; 2) average local $F \times F$ pixels and then down-sample the image by a factor of $F$.

\section{Gradient operator selection}

TABLE III. SROCC VALUES USING THREE GRADIENT OPERATORS

\begin{tabular}{cc}
\hline Database & SROCC \\
\hline Sobel & 0.8797 \\
Prewitt & 0.8776 \\
Scharr & 0.8825 \\
\hline
\end{tabular}

In our proposed IQA metrics FSIM/FSIM , the gradient magnitude (GM) needs to be calculated. To this end, three commonly used gradient operators listed in Table I were examined, and the one providing the best result was selected. Such a gradient operator selection process was carried out by assuming that all the parameters discussed in Section IV-B were fixed. The selection criterion was also that the gradient operator leading to a higher SROCC would be selected. The sub-dataset used in Section IV-B was used here. The SROCC values obtained by the three gradient operators on the tuning dataset are listed in Table III, from which we can see that the Scharr operator could achieve slightly better performance than the other two. Thus, in all of the following experiments, the Scharr operator was used to calculate the gradient in FSIM/FSIM $C$.

\section{Example to demonstrate the effectiveness of FSIM/FSIM ${ }_{C}$}

In this subsection, we use an example to demonstrate the effectiveness of FSIM/FSIM ${ }_{C}$ in evaluating the perceptible image quality. Fig. 4a is the I17 reference image in the TID2008 database, and Figs. 4b $\sim 4 \mathrm{f}$ show five distorted images of I17: I17_01_2, I17_03_2, I17_09_1, I17_11_2, and I17_12_2. Distortion types of Figs. $4 \mathrm{~b} \sim 4 \mathrm{f}$ are "additive Gaussian noise", "spatially correlated noise", "image denoising", "JPEG 2000 compression", and "JPEG transformation errors", respectively. According to the naming convention of TID2008, the last number (the last digit) of the image's name represents the distortion degree, and a greater number indicates a severer distortion. We compute the image quality of Figs. $4 \mathrm{~b} \sim 4 \mathrm{f}$ using various IQA metrics and the results are summarized in Table IV. We also list the subjective scores (extracted from 
TID2008) of these 5 images in Table IV. For each IQA metric and the subjective evaluation, higher scores mean higher image quality.

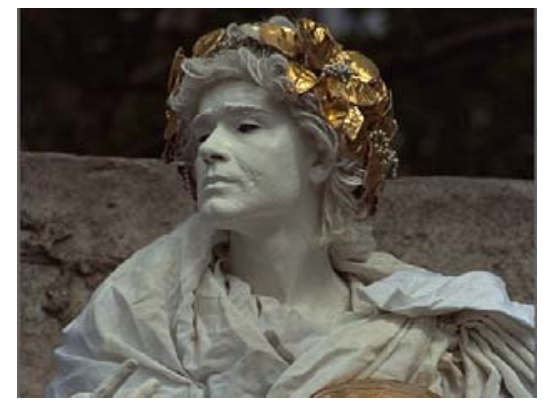

(a)

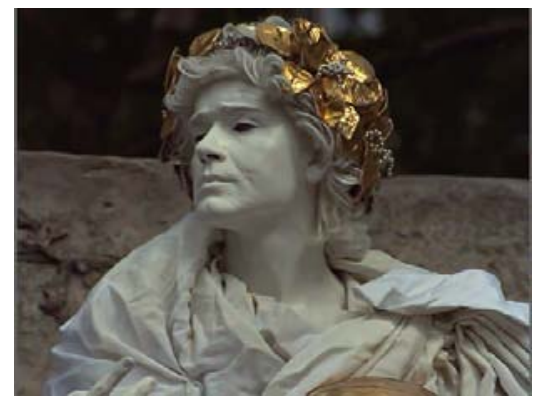

(d)

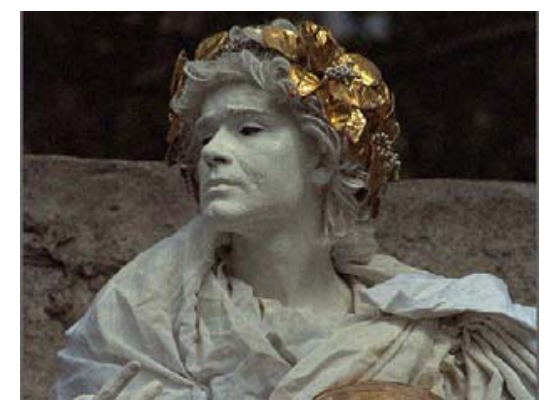

(b)

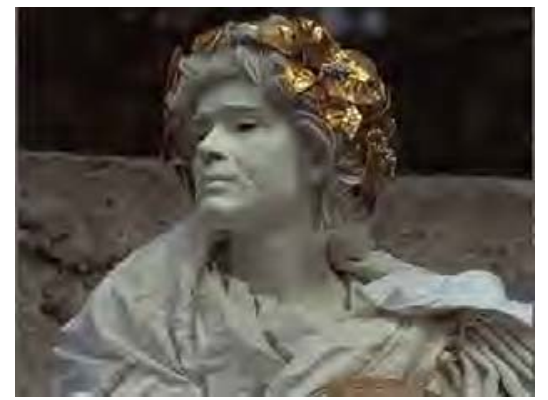

(e)

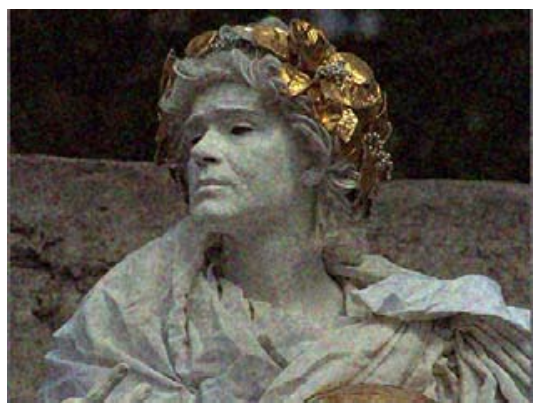

(c)

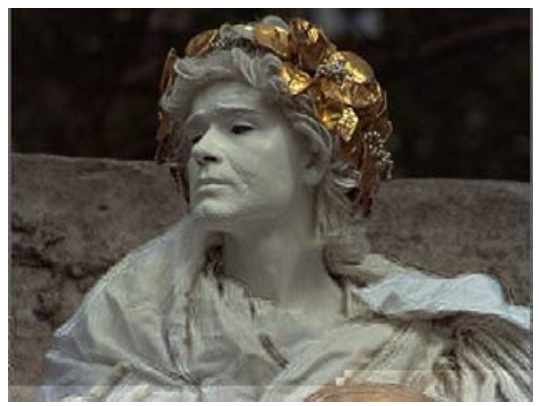

(f)

Fig. 4: (a) A reference image; (b) (f) are the distorted versions of (a) in the TID2008 database. Distortion types of (b) (f) are "additive Gaussian noise", "spatially correlated noise", "image denoising", "JPEG 2000 compression", and "JPEG transformation errors", respectively.

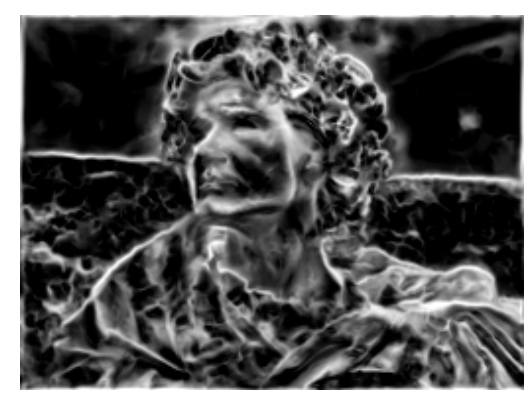

(a)

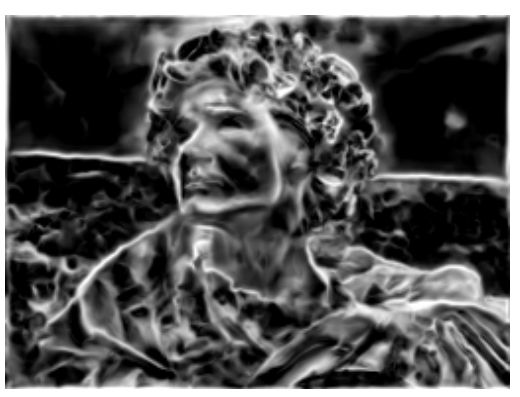

(d)

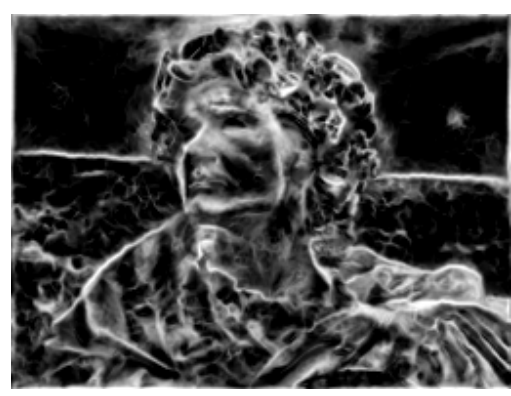

(b)

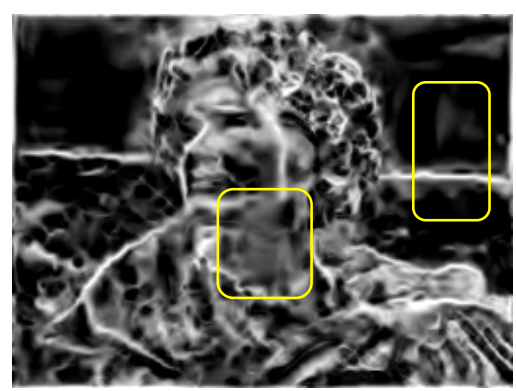

(e)

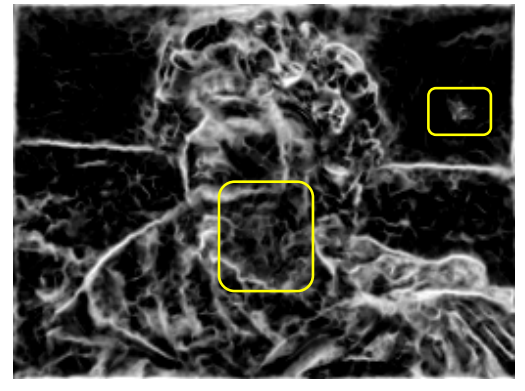

(c)

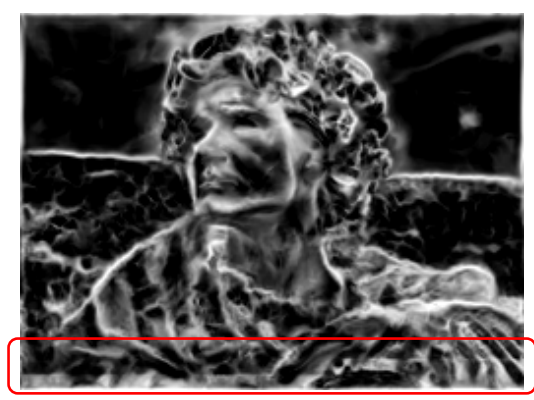

(f)

Fig. 5: (a) (f) are PC maps extracted from images Figs. 4a $\sim 4 f$, respectively. (a) is the PC map of the reference image while (b) (f) are the PC maps of the distorted images. (b) and (d) are more similar to (a) than (c), (e), and (f). In (c), (e), and (f), regions with obvious differences to the corresponding regions in (a) are marked by colorful rectangles. 
In order to show the correlation of each IQA metric with the subjective evaluation more clearly, in Table $\mathrm{V}$, we rank the images according to their quality scores computed by each metric as well as the subjective

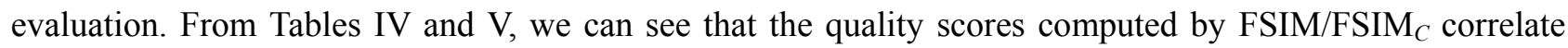
with the subjective evaluation much better than the other IQA metrics. From Table V we can also see that other than the proposed FSIM/FSIM ${ }_{C}$ metrics, all the other IQA metrics cannot give the same ranking as the subjective evaluations.

TABLE IV. QUALITY EVALUATION OF IAMGES IN FIG. 4

\begin{tabular}{cccccc}
\hline & Fig. 4b & Fig. 4c & Fig. 4d & Fig. 4e & Fig. 4f \\
\hline Subjective score & 5.2222 & 4.0571 & 6.1389 & 3.3429 & 5.2000 \\
FSIM & 0.9776 & 0.9281 & 0.9827 & 0.9085 & 0.9583 \\
FSIM $_{C}$ & 0.9741 & 0.9195 & 0.9817 & 0.9071 & 0.9582 \\
MS-SSIM & 0.9590 & 0.9109 & 0.9832 & 0.9170 & 0.9633 \\
VIF & 0.5803 & 0.4037 & 0.5670 & 0.1876 & 0.6484 \\
SSIM & 0.9268 & 0.8419 & 0.9686 & 0.8464 & 0.9306 \\
IFC & 3.5416 & 2.3441 & 3.9036 & 1.1710 & 8.0318 \\
VSNR & 30.7669 & 22.6702 & 32.2891 & 21.1772 & 18.6739 \\
NQM & 29.5608 & 21.0153 & 29.4932 & 19.4725 & 16.3083 \\
[21] & 0.8790 & 0.7158 & 0.9110 & 0.6503 & 0.9172 \\
PSNR & 27.1845 & 27.1577 & 34.0126 & 27.0330 & 26.9246 \\
\hline
\end{tabular}

TABLE V. RANKING OF IMAGES ACCORDING TO THEIR QUALITY COMPUTED BY EACH IQA METRIC

\begin{tabular}{cccccc}
\hline & Fig. 4b & Fig. 4c & Fig. 4d & Fig. 4e & Fig. 4f \\
\hline Subjective score & 2 & 4 & 1 & 5 & 3 \\
FSIM & 2 & 4 & 1 & 5 & 3 \\
FSIM $_{C}$ & 2 & 4 & 1 & 5 & 3 \\
MS-SSIM & 3 & 5 & 1 & 4 & 2 \\
VIF & 2 & 4 & 3 & 5 & 1 \\
SSIM & 3 & 5 & 1 & 4 & 2 \\
IFC & 3 & 4 & 2 & 5 & 1 \\
VSNR & 2 & 3 & 1 & 4 & 5 \\
NQM & 1 & 3 & 2 & 4 & 5 \\
[21] & 3 & 4 & 2 & 5 & 1 \\
PSNR & 2 & 3 & 1 & 4 & 5 \\
\hline
\end{tabular}

The success of FSIM/FSIM $C$ actually owes to the proper use of PC maps. Figs. $5 \mathrm{a} \sim 5 \mathrm{f}$ show the PC maps of the images in Figs. $4 \mathrm{a} \sim 4 \mathrm{f}$, respectively. We can see that images in Figs. $4 \mathrm{~b}$ and $4 \mathrm{~d}$ have better perceptible qualities than those in Figs. 4c, 4e, and 4f; meanwhile, by visually examination we can see that maps in Figs. 5b and 5d (PC maps of images in Figs. 4b and 4d) are more similar to the map in Fig. 5a (PC 
map of the reference image in Fig. 4a) than the maps in Figs. 5c, 5e, and 5f (PC maps of images in Figs. 4c, 4e, and 4f). In order to facilitate visual examination, in Figs. 5c, 5e, and 5f, regions with obvious differences to the corresponding regions in Fig. 5a are marked by rectangles. For example, in Fig. 5c, the neck region marked by the yellow rectangle has a perceptible difference to the same region in Fig. 5a. This example clearly illustrates that images of higher quality will have more similar PC maps to that of the reference image than images of lower quality. Therefore, by properly making use of PC maps in FSIM/FSIM $C$, we can predict the image quality consistently with human subjective evaluations. More statistically convincing results will be presented in the next two sub-sections.

\section{E. Overall performance comparison}

TABLE VI: PERFORMANCE COMPARISON OF IQA METRICS ON 6 BENCHMARK DATABASES

\begin{tabular}{|c|c|c|c|c|c|c|c|c|c|c|c|}
\hline & & SIM & $\operatorname{SIM}_{C}$ & 1S-SSIM & VIF & SSIM & IFC & VSNR & NQM & [21] & PSNR \\
\hline & SROCC & 805 & 0.8840 & 28 & 6 & 9 & 2 & & & $8 \pi$ & 5245 \\
\hline TID & KRC & & & & & & & & & & 696 \\
\hline \multirow[t]{2}{*}{2008} & PLCC & 0.8738 & 0.8762 & 0.8425 & 8090 & 0.7732 & 0.7359 & 0.6820 & 6135 & .7679 & 0.5309 \\
\hline & & 0.6525 & 0.6468 & 0.7299 & .7888 & 0.8511 & 0.9086 & 0.9815 & 1.0598 & 0.8595 & 1.1372 \\
\hline \multirow{4}{*}{ CSIQ } & SROCC & 9242 & 9310 & 0.9138 & 9193 & 0.8756 & 0.7482 & 0.8106 & 0.7402 & 0.7642 & 0.8057 \\
\hline & KROCC & 0.7 & 0 & & 7534 & 0. & 0.5 & 47 & 38 & 311 & 080 \\
\hline & PLCC & & & & 9277 & 0.8 & 0.8381 & & & 222 & 001 \\
\hline & RMSE & 0.1077 & & & 0980 & 0.1334 & & & 56 & 1494 & 575 \\
\hline \multirow{4}{*}{ LIVE } & SROCC & 0.9634 & 9645 & & 9631 & 0.9479 & 0.9234 & & & 0.8650 & 0.8755 \\
\hline & KROCC & 0.8337 & & & 8270 & 0.7963 & 0.7540 & & 413 & 0.6781 & 0.6864 \\
\hline & & 0.9 & 3 & 30 & 0.9598 & 0.9449 & 0.9248 & & & 765 & 0.8721 \\
\hline & & & & & 6734 & 8.9 & 10.392 & & & & 368 \\
\hline \multirow{4}{*}{ IVC } & $\mathrm{CC}$ & & & & 3966 & 0. & 78 & & & 83 & 385 \\
\hline & KROCC & 64 & & & 7165 & 0.7223 & 0.7192 & & & 441 & 220 \\
\hline & PLCC & 0.9376 & 0.9392 & & 0.9028 & 0.9119 & 0.9080 & & & 0.8454 & 0.7199 \\
\hline & & & & & .5239 & 0.4999 & 0.5105 & & & .6507 & 456 \\
\hline \multirow{4}{*}{ MICT } & & & & & & & & & & 923 & 130 \\
\hline & KROCC & 2 & & & 329 & 0.6 & 0.6 & & & 52 & 447 \\
\hline & PLCC & 0.9078 & 75 & & 0.9144 & 0.8887 & 0.8434 & & 55 & 0.7208 & 0.6426 \\
\hline & RMSE & 0.5248 & 5257 & & 0.5066 & 0.5738 & 0.6723 & 0.6147 & 569 & 0.8674 & 0.9588 \\
\hline \multirow{4}{*}{ A57 } & $\mathrm{OOCC}$ & 9181 & - & & .6223 & 0.8066 & 0.3185 & & & 0.7155 & 189 \\
\hline & $\mathrm{CC}$ & 39 & - & & 0.4589 & 0.6058 & 0.2378 & & & 0.5275 & 309 \\
\hline & & & - & & & & & & & 0.7399 & \\
\hline & RMSE & 0.0933 & - & 0.1293 & 0.1936 & 0.1469 & 0.2189 & 0.0781 & 0.1468 & 0.1653 & 0.1849 \\
\hline
\end{tabular}

In this section, we compare the general performance of the competing IQA metrics. Table VI lists the SROCC, KROCC, PLCC, and RMSE results of FSIM/FSIM ${ }_{C}$ and the other 8 IQA algorithms on the TID2008, CSIQ, LIVE, IVC, MICT, and A57 databases. For each performance measure, the three IQA indices producing the best results are highlighted in boldface for each database. It should be noted that except for $\mathrm{FSIM}_{C}$, all the other IQA indices are based on the luminance component of the image. From Table 
VI, we can see that the proposed feature-similarity based IQA metric FSIM or FSIM $_{C}$ performs consistently well across all the databases. In order to demonstrate this consistency more clearly, in Table VII we list the

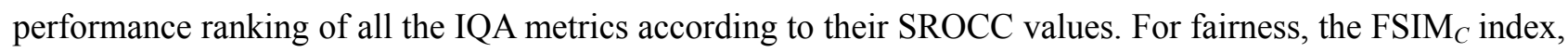
which also exploits the chrominance information of images, is excluded in Table VII.

\section{TABLE VII: RANKING OF IQA METRICS' PERFORMANCE (EXCEPT FOR FSIM ${ }_{C}$ ) ON SIX DATABASES}

\begin{tabular}{ccccccc}
\hline & TID2008 & CSIQ & LIVE & IVC & MICT & A57 \\
\hline FSIM & $\mathbf{1}$ & $\mathbf{1}$ & $\mathbf{1}$ & $\mathbf{1}$ & 2 & 2 \\
MS-SSIM & 2 & 3 & 4 & 5 & 4 & 3 \\
VIF & 4 & 2 & 2 & 4 & $\mathbf{1}$ & 7 \\
SSIM & 3 & 4 & 3 & 2 & 5 & 4 \\
IFC & 8 & 8 & 6 & 3 & 7 & 9 \\
VSNR & 6 & 5 & 5 & 8 & 6 & $\mathbf{1}$ \\
NQM & 7 & 9 & 7 & 7 & 3 & 5 \\
[21] & 5 & 7 & 9 & 6 & 8 & 6 \\
PSNR & 9 & 6 & 8 & 9 & 9 & 8 \\
\hline
\end{tabular}

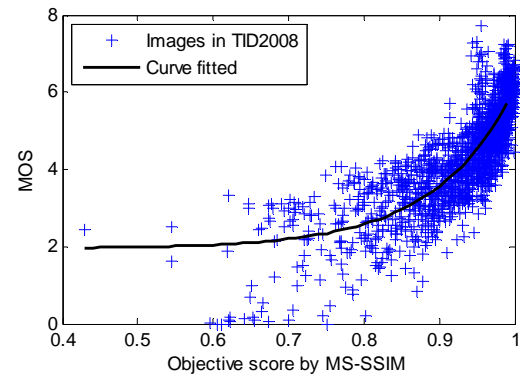

(a)

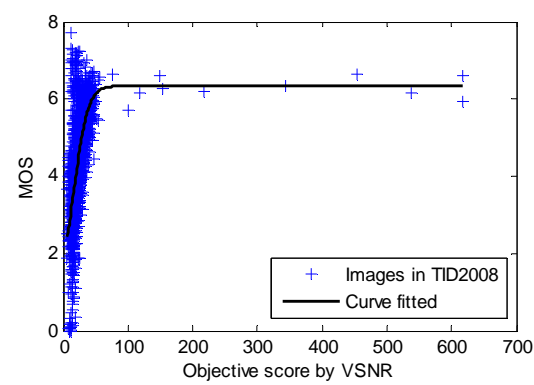

(d)

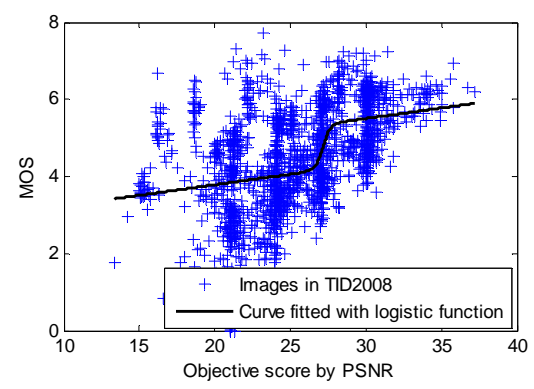

(g)

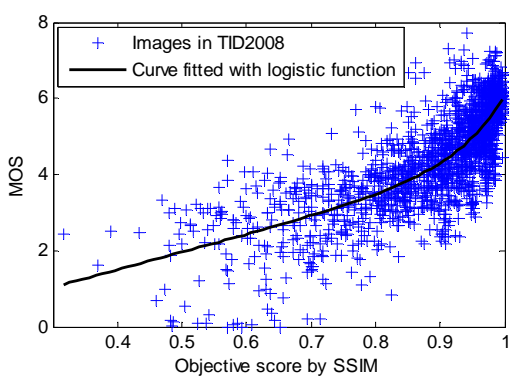

(b)

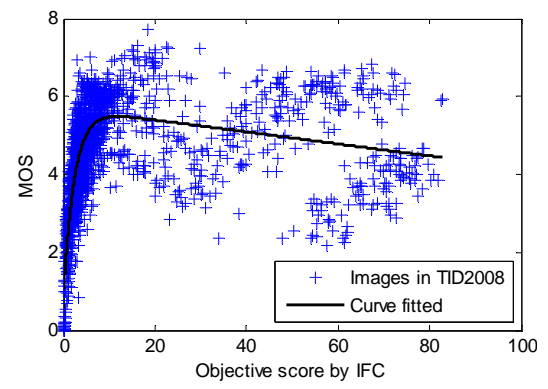

(e)

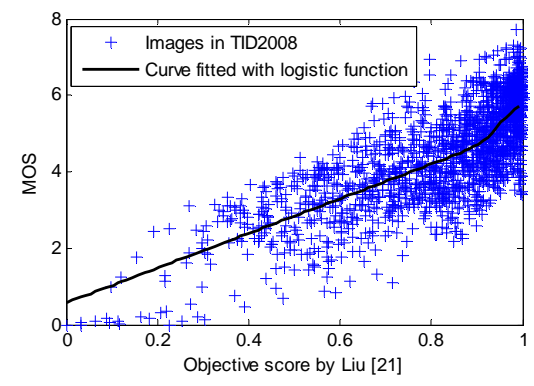

(h)

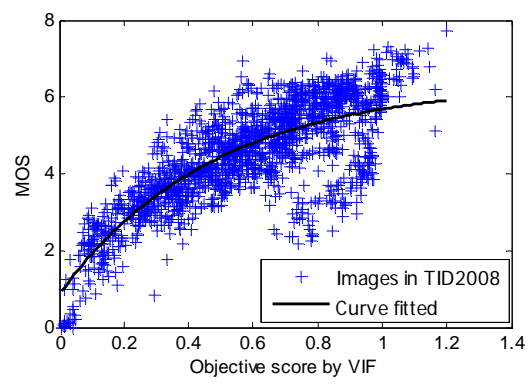

(c)

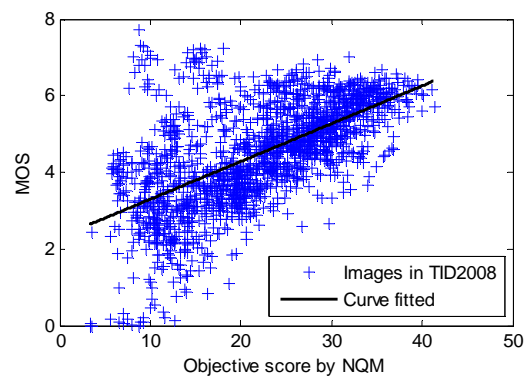

(f)

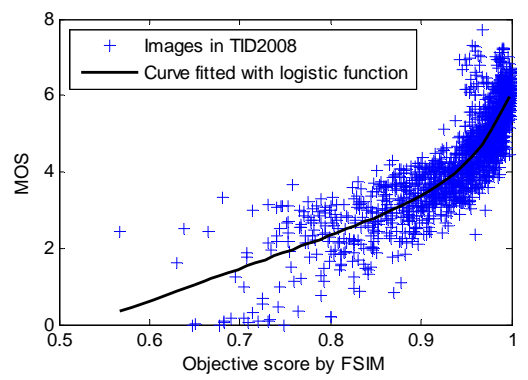

(i)

Fig. 6: Scatter plots of subjective MOS versus scores obtained by model prediction on the TID 2008 database. (a) MS-SSIM; (b) SSIM; (c) VIF; (d) VSNR; (e) IFC; (f) NQM; (g) PSNR; (h) method in [21] and (i) FSIM. 
From the experimental results summarized in Table VI and Table VII, we can see that our methods achieve the best results on almost all the databases, except for MICT and A57. Even on these two databases,

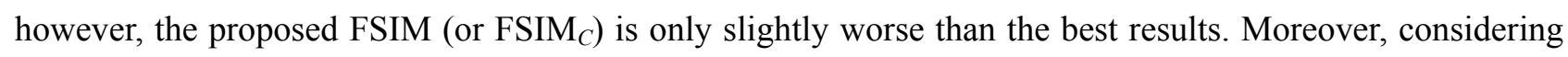
the scales of the databases, including the number of images, the number of distortion types, and the number of observers, we think that the results obtained on TID2008, CSIQ, LIVE and IVC are much more convincing than those obtained on MICT and A57. Overall speaking, FSIM and FSIM $_{C}$ achieve the most consistent and stable performance across all the 6 databases. By contrast, for the other methods, they may work well on some databases but fail to provide good results on other databases. For example, although VIF can get very pleasing results on LIVE, it performs poorly on TID2008 and A57. The experimental results also demonstrate that the chromatic information of an image does affect its perceptible quality since FSIM $_{C}$ has better performance than FSIM on all color image databases. Fig. 6 shows the scatter distributions of subjective MOS versus the predicted scores by FSIM and the other 8 IQA indices on the TID 2008 database. The curves shown in Fig. 6 were obtained by a nonlinear fitting according to Eq. (12). From Fig. 6, one can see that the objective scores predicted by FSIM correlate much more consistently with the subjective evaluations than the other methods.

\section{F. Performance on individual distortion types}

In this experiment, we examined the performance of the competing methods on different image distortion types. We used the SROCC score, which is a widely accepted and used evaluation measure for IQA metrics $[1,39]$, as the evaluation measure. By using the other measures, such as KROCC, PLCC, and RMSE, similar conclusions could be drawn. The three largest databases, TID2008, CSIQ and LIVE, were used in this experiment. The experimental results are summarized in Table VIII. For each database and each distortion type, the first 3 IQA indices producing the highest SROCC values are highlighted in boldface. We can have some observations based on the results listed in Table VIII. In general, when the distortion type is known beforehand, FSIM $_{C}$ performs the best, while FSIM and VIF have comparable performance. FSIM, FSIM $C$ and VIF perform much better than the other IQA indices. Compared with VIF, FSIM and FSIM $C$ are more capable in dealing with the distortions of "denoising", "quantization noise", and "mean shift". By contrast, for the distortions of "masked noise" and "impulse noise", VIF performs better than FSIM and FSIM . 
Moreover, results in Table VIII once again corroborates that the chromatic information does affect the perceptible quality since $\mathrm{FSIM}_{C}$ has better performance than FSIM on each database for nearly all the distortion types.

TABLE VIII: SROCC VALUES OF IQA METRICS FOR EACH DISTORTION TYPE

\begin{tabular}{|c|c|c|c|c|c|c|c|c|c|c|c|}
\hline & & FSIM & FSIM $_{C}$ & MS- & VIF & SIM & IFC & VSNR & NQM & {$[21]$} & PSNR \\
\hline \multirow{17}{*}{$\begin{array}{c}\text { TID } \\
2008\end{array}$} & awgn & 0.8566 & 0.8758 & 0.8094 & 0.8799 & 0.8107 & 0.5817 & 0.7728 & 0.7679 & 0.5069 & 0.9114 \\
\hline & awgn-color & 0.8527 & 0.8931 & 0.8064 & 0.8785 & 0.8029 & 0.5528 & 0.7793 & 0.7490 & 0.4625 & 0.9068 \\
\hline & spatial corr-noise & 0.8483 & 0.8711 & 0.8195 & 0.8703 & 0.8144 & 0.5984 & 0.7665 & 0.7720 & 0.6065 & 0.9229 \\
\hline & masked noise & 0.8021 & 0.8264 & 0.8155 & 0.8698 & 0.7795 & 0.7326 & 0.7295 & 0.7067 & 0.5301 & 0.8487 \\
\hline & high-fre-noise & 0.9093 & 0.9156 & 0.8685 & 0.9075 & 0.8729 & 0.7361 & 0.8811 & 0.9015 & 0.6935 & 0.9323 \\
\hline & impulse 1 & 0.7452 & 0.7719 & 0.6868 & 0.8331 & 0.6732 & 0.5334 & 0.6471 & 0.7616 & 0.4537 & 0.9177 \\
\hline & quantization noise & 0.8564 & 0.8726 & 0.8537 & 0.7956 & 0.8531 & 0.5911 & 0.8270 & 0.8209 & 0.6214 & 0.8699 \\
\hline & blur & 0.9472 & 0.9472 & 0.9607 & 0.9546 & 0.9544 & 0.8766 & 0.9330 & 0.8846 & 0.8883 & 0.8682 \\
\hline & denoising & 0.9603 & 0.9618 & 0.9571 & 0.9189 & 0.9530 & 0.8002 & 0.9286 & 0.9450 & 0.7878 & 0.9381 \\
\hline & jpg-comp & 0.9279 & 0.9294 & 0.9348 & 0.9170 & 0.9252 & 0.8181 & 0.9174 & 0.9075 & 0.8186 & 0.9011 \\
\hline & jpg2k-comp & 0.9773 & 0.9780 & 0.9736 & 0.9713 & 0.9625 & 0.9445 & 0.9515 & 0.9532 & 0.9301 & 0.8300 \\
\hline & jpg-trans-error & 0.8708 & 0.8756 & 0.8736 & 0.8582 & 0.8678 & 0.7966 & 0.8056 & 0.7373 & 0.8334 & 0.7665 \\
\hline & jpg2k-trans-error & 0.8544 & 0.8555 & 0.8 & 0.8510 & 0.8577 & 0.7303 & 0.7909 & 0.7262 & 0.7164 & 0.7765 \\
\hline & pattern-noise & 0.7491 & 0.7514 & 0.7336 & 0.7608 & 0.7107 & 0.8410 & 0.5716 & 0.6800 & 0.7677 & 0.5931 \\
\hline & block-distortion & 0.8492 & 0.8464 & 0.7617 & 0.8320 & 0.8462 & 0.6767 & 0.1926 & 0.2348 & 0.7282 & 0.5852 \\
\hline & mean shift & 0.6720 & 0.6554 & 0.7374 & 0.5132 & 0.7231 & 0.4375 & 0.3715 & 0.5245 & 0.3487 & 0.6974 \\
\hline & contrast & 0.6481 & 0.6510 & 0.6400 & 0.8190 & 0.5246 & 0.2748 & 0.4239 & 0.6191 & 0.3883 & 0.6126 \\
\hline \multirow{6}{*}{ CSIQ } & awgn & 0.9262 & 0.9359 & 0.9471 & 0.9571 & 0.8974 & 0.8460 & 0.9241 & 0.9384 & 0.7501 & 0.9363 \\
\hline & jpg-comp & 0.9654 & 0.9664 & 0.9622 & 0.9705 & 0.9546 & 0.9395 & 0.9036 & 0.9527 & 0.9088 & 0.8882 \\
\hline & jpg2k-comp & 0.9685 & 0.9704 & 0.9691 & 0.9672 & 0.9606 & 0.9262 & 0.9480 & 0.9631 & 0.8886 & 0.9363 \\
\hline & $1 /$ f noise & 0.9234 & 0.9370 & 0.9330 & 0.9509 & 0.8922 & 0.8279 & 0.9084 & 0.9119 & 0.7905 & 0.9338 \\
\hline & blur & 0.9729 & 0.9729 & 0.9720 & 0.9747 & 0.9609 & 0.9593 & 0.9446 & 0.9584 & 0.9551 & 0.9289 \\
\hline & contrast & 0.9420 & 0.9438 & 0.9521 & 0.9361 & 0.7922 & 0.5416 & 0.8700 & 0.9479 & 0.4326 & 0.8622 \\
\hline \multirow{5}{*}{ LIVE } & jpg2k-comp & 0.9717 & 0.9724 & 0.9654 & 0.9683 & 0.9614 & 0.9100 & 0.9551 & 0.9435 & 0.8533 & 0.8954 \\
\hline & jpg-comp & 0.9834 & 0.9840 & 0.9793 & 0.9842 & 0.9764 & 0.9440 & 0.9657 & 0.9647 & 0.9127 & 0.8809 \\
\hline & awgn & 0.9652 & 0.9716 & 0.9731 & 0.9845 & 0.9694 & 0.9377 & 0.9785 & 0.9863 & 0.9079 & 0.9854 \\
\hline & blur & 0.9708 & 0.9708 & 0.9584 & 0.9722 & 0.9517 & 0.9649 & 0.9413 & 0.8397 & 0.9365 & 0.7823 \\
\hline & jpg2k-trans-error & 0.9499 & 0.9519 & 0.9321 & 0.9652 & 0.9556 & 0.9644 & 0.9027 & 0.8147 & 0.8765 & 0.8907 \\
\hline
\end{tabular}

\section{CONCLUSIONS}

In this paper, we proposed a novel low-level feature based image quality assessment (IQA) metric, namely Feature-SIMilarity (FSIM) index. The underlying principle of FSIM is that HVS perceives an image mainly 
based on its salient low-level features. Specifically, two kinds of features, the phase congruency (PC) and the gradient magnitude (GM), are used in FSIM, and they represent complementary aspects of the image visual quality. The PC value is also used to weight the contribution of each point to the overall similarity of two images. We then extended FSIM to FSIM $C$ by incorporating the image chromatic features into consideration. The FSIM and FSIM ${ }_{C}$ indices were compared with eight representative and prominent IQA metrics on six

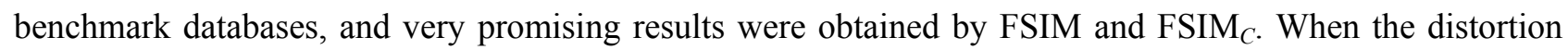
type is known beforehand, FSIM $_{C}$ performs the best while FSIM achieves comparable performance with VIF. When all the distortion types are involved (i.e. all the images in a test database are used), FSIM and FSIM $C$ outperform all the other IQA metrics used in comparison. Particularly, they perform consistently well across all the test databases, validating that they are very robust IQA metrics.

\section{REFERENCES}

[1] Z. Wang, A.C. Bovik, H.R. Sheikh, and E.P. Simoncelli, "Image quality assessment: from error visibility to structural similarity”, IEEE Trans. Image Process., vol. 13, no. 4, pp. 600-612, Apr. 2004.

[2] N. Damera-Venkata, T.D. Kite, W.S. Geisler, B.L. Evans, and A.C. Bovik, "Image quality assessment based on a degradation model”, IEEE Trans. Image Process., vol. 9, no. 4, pp. 636-650, Apr. 2000.

[3] D.M. Chandler and S.S. Hemami, "VSNR: a wavelet-based visual signal-to-noise ratio for natural images", IEEE Trans. Image Process., vol. 16, no. 9, pp. 2284-2298, Sep. 2007.

[4] H.R. Sheikh and A.C. Bovik, "Image information and visual quality", IEEE Trans. Image Process., vol. 15, no. 2, pp. 430-444, Feb. 2006.

[5] Z. Wang, E.P. Simoncelli, and A.C. Bovik, "Multi-scale structural similarity for image quality assessment", presented at the IEEE Asilomar Conf. Signals, Systems and Computers, Nov. 2003.

[6] C. Li and A.C. Bovik, “Three-component weighted structural similarity index”, in Proc. SPIE, vol. $7242,2009$.

[7] H.R. Sheikh, A.C. Bovik, and G. de Veciana, "An information fidelity criterion for image quality assessment using natural scene statistics”, IEEE Trans. Image Process., vol. 14, no. 12, pp. 2117-2128, Dec. 2005.

[8] M.P. Sampat, Z. Wang, S. Gupta, A.C. Bovik, and M.K. Markey, “Complex wavelet structural similarity: a new image similarity index”, IEEE Trans. Image Process., vol. 18, no. 11, pp. 2385-2401, Nov. 2009.

[9] H.R. Sheikh, M.F. Sabir, and A.C. Bovik, "A statistical evaluation of recent full reference image quality assessment algorithms", IEEE Trans. Image Process., vol. 15, no. 11, pp. 3440-3451, Nov. 2006. 
[10] N. Ponomarenko, V. Lukin, A. Zelensky, K. Egiazarian, M. Carli, and F. Battisti, “TID2008 - A database for evaluation of full-reference visual quality assessment metrics", Advances of Modern Radioelectronics, vol. 10, pp. $30-45,2009$.

[11]Z. Wang and Q. Li, “Information content weighting for perceptual image quality assessment", IEEE Trans. Image Process., accepted.

[12] E.C. Larson and D.M. Chandler, "Unveiling relationships between regions of interest and image fidelity metrics", in Proc. SPIE Visual Comm. and Image Process., vol. 6822, pp. 6822A1-16, Jan. 2008.

[13]E.C. Larson, C. Vu, and D.M. Chandler, “Can visual fixation patterns improve image fidelity assessment?”, in Proc. IEEE Int. Conf. Image Process., 2008, pp. 2572-2575.

[14] D. Marr, Vision. New York: W. H. Freeman and Company, 1980.

[15] D. Marr and E. Hildreth, “Theory of edge detection”, Proc. R. Soc. Lond. B, vol. 207, no. 1167, pp. 187-217, Feb. 1980.

[16] M.C. Morrone and D.C. Burr, "Feature detection in human vision: a phase-dependent energy model", Proc. R. Soc. Lond. B, vol. 235, no. 1280, pp. 221-245, Dec. 1988.

[17] M.C. Morrone, J. Ross, D.C. Burr, and R. Owens, "Mach bands are phase dependent”, Nature, vol. 324, no. 6049, pp. 250-253, Nov. 1986.

[18] M.C. Morrone and R.A. Owens, "Feature detection from local energy", Pattern Recognit. Letters, vol. 6, no. 5, pp. 303-313, Dec. 1987.

[19] P. Kovesi, “Image features from phase congruency”, Videre: J. Comp. Vis. Res., vol. 1, no. 3, pp. 1-26, 1999.

[20] L. Henriksson, A. Hyvärinen, and S. Vanni, "Representation of cross-frequency spatial phase relationships in human visual cortex", J. Neuroscience, vol. 29, no. 45, pp. 14342-14351, Nov. 2009.

[21]Z. Liu and R. Laganière, "Phase congruence measurement for image similarity assessment", Pattern Recognit. Letters, vol. 28, no. 1, pp. 166-172, Jan. 2007.

[22]Z. Wang and E.P. Simoncelli, "Local phase coherence and the perception of blur", in Adv. Neural Information Processing Systems., 2004, pp. 786-792.

[23] R. Hassen, Z. Wang, and M. Salama, "No-reference image sharpness assessment based on local phase coherence measurement", in Proc. IEEE Int. Conf. Acoust., Speech, and Signal Processing, 2010, pp. 2434-2437.

[24]D. Gabor, “Theory of communication”, J. Inst. Elec. Eng., vol. 93, no. III, pp. 429-457, 1946.

[25]D. J. Field, "Relations between the statistics of natural images and the response properties of cortical cells", J. Opt. Soc. Am. A, vol. 4, no. 12, pp. 2379-2394, Dec. 1987. 
[26] C. Mancas-Thillou and B. Gosselin, "Character segmentation-by-recognition using log-Gabor filters", in Proc. Int. Conf. Pattern Recognit., 2006, pp. 901-904.

[27] S. Fischer, F. Šroubek, L. Perrinet, R. Redondo, and G. Cristóbal, “Self-invertible 2D log-Gabor wavelets”, Int. J. Computer Vision, vol. 75, no. 2, pp. 231-246, Nov. 2007.

[28] W. Wang, J. Li, F. Huang, and H. Feng, "Design and implementation of log-Gabor filter in fingerprint image enhancement", Pattern Recognit. Letters, vol. 29, no. 3, pp. 301-308, Feb. 2008.

[29] R. Jain, R. Kasturi, and B.G. Schunck, Machine Vision. McGraw-Hill, Inc, 1995.

[30]B. Jähne, H. Haubecker, and P. Geibler, Handbook of Computer Vision and Applications. Academic Press, 1999.

[31]C. Yang and S.H. Kwok, "Efficient gamut clipping for color image processing using LHS and YIQ", Optical Engineering, vol. 42, no. 3, pp.701-711, Mar. 2003.

[32] E.C. Larson and D.M. Chandler, “Categorical Image Quality (CSIQ) Database”, http://vision.okstate.edu/csiq.

[33]H.R. Sheikh, K. Seshadrinathan, A.K. Moorthy, Z. Wang, A.C. Bovik, and L.K. Cormack, "Image and video quality assessment research at LIVE", http://live.ece.utexas.edu/research/quality.

[34]A. Ninassi, P. Le Callet, and F. Autrusseau, "Subjective quality assessment-IVC database", http://www2.irccyn.ec-nantes.fr/ivcdb.

[35] Y. Horita, K. Shibata, Y. Kawayoke, and Z.M. Parves Sazzad, "MICT Image Quality Evaluation Database", http://mict.eng.u-toyama.ac.jp/mict/index2.html.

[36] D.M. Chandler and S.S. Hemami, “A57 database”, http://foulard.ece.cornell.edu/dmc27/vsnr/vsnr.html.

[37]Z. Wang, “SSIM Index for Image Quality Assessment”, http://www.ece.uwaterloo.ca/ Z70wang/research/ssim/.

[38]M. Gaubatz and S.S. Hemami, "MeTriX MuX Visual Quality Assessment Package", http://foulard.ece.cornell.edu/gaubatz/metrix_mux.

[39] VQEG, "Final report from the video quality experts group on the validation of objective models of video quality assessment", http://www.vqeg.org, 2000. 